\title{
Electronic Nature of Charge Density Wave and Electron-Phonon Coupling in Kagome Superconductor KV3Sb5
}

\section{Xingjiang Zhou ( $\nabla$ xjzhou@iphy.ac.cn )}

National Lab for Superconductivity, Institute of Physics, Chinese Academy of Sciences https://orcid.org/0000-0002-5261-1386

\section{Hai-Lan Luo}

Institute of Physics, Chinese Academy of Sciences, Beijing 100190, China https://orcid.org/0000-00029925-0450

\section{Qiang Gao}

Institute of Physics

\section{Hongxiong Liu}

Institute of Physics, Chinese Academy of Sciences

\section{Yuhao Gu}

Institute of Physics, Chinese Academy of Sciences

\section{Ding-Song Wu}

Institute of Physics

\section{Changjiang Yi}

Beijing National Laboratory for Condensed Matter Physics and Institute of Physics, Chinese Academy of

Sciences

Junjie Jia

Beijing National Laboratory for Condensed Matter Physics, Institute of Physics, Chinese Academy of

Sciences

\section{Shilong Wu}

Institute of Physics, Chinese Academy of Sciences

\section{Xiangyu Luo}

Beijing National Laboratory for Condensed Matter Physics, Institute of Physics, Chinese Academy of

\section{Sciences}

\section{$\mathrm{Yu} \mathrm{Xu}$}

Chinese Academy of Sciences

\section{Lin Zhao}

Chinese Academy of Sciences

\section{Qing-Yan Wang}

Institute of Physics, Chinese Academy of Sciences https://orcid.org/0000-0002-1179-0293 


\section{Hanqing Mao}

Institute of Physics, Chinese Academy of Sciences

\section{Guodong Liu}

Chinese Academy of Sciences

\section{Zhihai Zhu}

Institute of Physics, Chinese Academy of Sciences

\section{Youguo Shi}

Institute of Physics, Chinese Academy of Sciences

Kun Jiang

Institute of Physics, Chinese Academy of Sciences

Jiangping $\mathrm{Hu}$

Institute of Physics

\section{Zuyan Xu}

Technical Institute of Physics and Chemistry

\section{Article}

Keywords: Kagome superconductors, charge density wave, KV3Sb5

Posted Date: August 3rd, 2021

DOI: https://doi.org/10.21203/rs.3.rs-690777/v1

License: (c) (1) This work is licensed under a Creative Commons Attribution 4.0 International License. Read Full License

Version of Record: A version of this preprint was published at Nature Communications on January 12th, 2022. See the published version at https://doi.org/10.1038/s41467-021-27946-6. 


\title{
1. Electronic Nature of Charge Density Wave and Electron-Phonon Coupling in Kagome Superconductor $\mathrm{KV}_{3} \mathrm{Sb}_{5}$
}

\author{
Hailan Luo ${ }^{1,2 \sharp}$, Qiang $\mathrm{Gao}^{1 \sharp}$, Hongxiong $\mathrm{Liu}^{1,2 \sharp}$, Yuhao $\mathrm{Gu}^{1 \sharp}$, Dingsong \\ $\mathrm{Wu}^{1,2}$, Changjiang $\mathrm{Yi}^{1}$, Junjie Jia ${ }^{1,2}$, Shilong $\mathrm{Wu}^{1}$, Xiangyu $\mathrm{Luo}^{1,2}$, Yu Xu${ }^{1}$, \\ Lin Zhao ${ }^{1}$, Qingyan Wang ${ }^{1}$, Hanqing $\mathrm{Mao}^{1}$, Guodong Liu ${ }^{1,2}$, Zhihai Zhu ${ }^{1}$, \\ Youguo Shi ${ }^{1 *}$, Kun Jiang ${ }^{1 *}$, Jiangping $\mathrm{Hu}^{1,2}$, Zuyan $\mathrm{Xu}^{3}$ and X. J. Zhou ${ }^{1,2,4,5 *}$ \\ ${ }^{1}$ Beijing National Laboratory for Condensed Matter Physics, \\ Institute of Physics, Chinese Academy of Sciences, Beijing 100190, China \\ ${ }^{2}$ University of Chinese Academy of Sciences, Beijing 100049, China \\ ${ }^{3}$ Technical Institute of Physics and Chemistry, \\ Chinese Academy of Sciences, Beijing 100190, China \\ ${ }^{4}$ Songshan Lake Materials Laboratory, Dongguan 523808, China \\ ${ }^{5}$ Beijing Academy of Quantum Information Sciences, Beijing 100193, China \\ ${ }^{\sharp}$ These people contributed equally to the present work. \\ *Corresponding authors: xjzhou@iphy.ac.cn, \\ ygshi@iphy.ac.cn and jiangkun@iphy.ac.cn
}

(Dated: July 15, 2021) 
The Kagome superconductors $\mathrm{AV}_{3} \mathrm{Sb}_{5}(\mathrm{~A}=\mathrm{K}, \mathrm{Rb}, \mathrm{Cs})$ have received enor19 mous attention due to their nontrivial topological electronic structure, anoma${ }_{20}$ lous physical properties and superconductivity. Unconventional charge density ${ }_{21}$ wave $(\mathrm{CDW})$ has been detected in $\mathrm{AV}_{3} \mathrm{Sb}_{5}$ that is found to be intimately inter${ }_{22}$ twined with the anomalous Hall effect and superconductivity. High-precision ${ }_{23}$ electronic structure determination is essential to understand the origin of the ${ }_{24}$ CDW transition and its interplay with electron correlation, topology and su${ }_{25}$ perconductivity, yet, little evidence has been found about the impact of the ${ }_{26} \mathrm{CDW}$ state on the electronic structure in $\mathrm{AV}_{3} \mathrm{Sb}_{5}$. Here we unveil electronic ${ }_{27}$ nature of the CDW phase in our high-resolution angle-resolved photoemission ${ }_{28}$ (ARPES) measurements on $\mathrm{KV}_{3} \mathrm{Sb}_{5}$. We have observed CDW-induced Fermi ${ }_{29}$ surface reconstruction and the associated band structure folding. The CDW30 induced band splitting and the associated gap opening have been revealed at the ${ }_{31}$ boundary of the pristine and reconstructed Brillouin zone. The Fermi surface${ }_{32}$ and momentum-dependent CDW gap is measured for the first time and the ${ }_{33}$ strongly anisotropic CDW gap is observed for all the V-derived Fermi surface ${ }_{34}$ sheets. In particular, we have observed signatures of the electron-phonon cou${ }_{35}$ pling for all the $\mathrm{V}$-derived bands. These results provide key insights in under${ }_{36}$ standing the nature of the CDW state and its interplay with superconductivity ${ }_{37}$ in $\mathrm{AV}_{3} \mathrm{Sb}_{5}$ superconductors.

38 The newly discovered Kagome superconductors $\mathrm{AV}_{3} \mathrm{Sb}_{5}(\mathrm{~A}=\mathrm{K}, \mathrm{Rb}, \mathrm{Cs})$ have attracted 39 much attention because they provide an ideal platform to investigate the interplay of topol40 ogy, electron correlation effects and superconductivity[1,2]. In the crystal structure of ${ }_{41} \mathrm{AV}_{3} \mathrm{Sb}_{5}$ (Fig. 1a), the vanadium atoms form a Kagome lattice that is a two-dimensional 42 network of corner-sharing triangles. The metallic Kagome lattice presents unique electronic ${ }_{43}$ structure characterized by a Dirac cone at the Brillouin zone corner, von Hove singularities ${ }_{44}$ (VHS) at the zone boundary and a flat band throughout the entire Brillouin zone[3, 4]. Such 45 a Kagome lattice is expected to harbour topological states[3, 5], fractional charges[4, 6], den${ }_{46}$ sity wave orders $[3,7,8]$ and unconventional superconductivity[8-11]. For example, $\mathrm{AV}_{3} \mathrm{Sb}_{5}$ ${ }_{47}$ family exhibit anomolous Hall effect[12, 13], although there is neither local-moment nor ${ }_{48}$ long-range magnetic ordering present in them[1, 12, 14]; unconventional charge density wave ${ }_{49}(\mathrm{CDW})$ has been revealed in $\mathrm{AV}_{3} \mathrm{Sb}_{5}[15-17]$. At present, the pairing symmetry of the $\mathrm{AV}_{3} \mathrm{Sb}_{5}$ 
50 superconductors has been extensively studied and it is still being debated whether the su51 perconductivity is unconventional[18-22].

52 The family of Kagome compounds $\mathrm{AV}_{3} \mathrm{Sb}_{5}(\mathrm{~A}=\mathrm{K}, \mathrm{Rb}, \mathrm{Cs})$ exhibit a $\mathrm{CDW}$ transition at ${ }_{53} 78 \sim 103 \mathrm{~K}$ observed by transport measurements[1, 2, 15-17, 23, 24]. Such a CDW transition 54 corresponds to a three-dimensional $2 \times 2 \times 2$ lattice reconstruction $[15,17,19]$ and promotes a ${ }_{55}$ structural distortion with three different V-V bond lengths named as Tri-Hexagonal ( $\left.\operatorname{TrH}\right)$ 56 structure (Fig. 1b) [15, 25]. The CDW state shows an unusual magnetic response[15] that 57 is intimately related to the anomalous Hall effect[13] and competes with superconductivity 58 under pressure[26-31]. Undestanding the electronic structure of the CDW state is essential 59 to reveal its nature and relation to the topological state and superconductivity[25, 32-36]. ${ }_{60}$ However, little is known about the impact of the CDW state on the electronic structure in ${ }_{61} \mathrm{AV}_{3} \mathrm{Sb}_{5}[37-39]$.

62 In this paper, we carried out high-resolution angle-resolved photoemission (ARPES) mea${ }_{63}$ surements to investigate the nature of the $\mathrm{CDW}$ instability in $\mathrm{KV}_{3} \mathrm{Sb}_{5}$. Clear evidence of ${ }_{64}$ electronic structure reconstruction induced by the $2 \times 2 \mathrm{CDW}$ transition is revealed by the 65 observation of the band and Fermi surface foldings. The band splitting and CDW gap 66 opening on multiple bands are observed at the boundaries of both the original and $2 \times 2$ ${ }_{67}$ reconstructed Brillouin zones. We have clearly resolved all the Fermi surface that enables ${ }_{68}$ us to map out the Fermi surface- and momentum-dependent CDW gap. The signature of ${ }_{69}$ electron-phonon coupling has been found on the V-derived bands. These results provide key 70 insight in understanding the origin of the CDW and its role on the exotic physical properties 71 and superconductivity in Kagome superconductors.

72 High-quality $\mathrm{KV}_{3} \mathrm{Sb}_{5}$ single crystals are prepared by a two-steps self-flux method[1] and 73 characterized by X-ray diffraction (see Methods and Fig. S1a in Supplementary Materials). 74 The transport and magnetic measurements show that our samples exhibit a CDW phase 75 transition at $T_{C D W} \sim 80 \mathrm{~K}$, consistent with the previous reports[1, 23]. In the normal state 76 above $T_{C D W}, \mathrm{KV}_{3} \mathrm{Sb}_{5}$ crystallizes in a hexagonal structure with the $P 6 / \mathrm{mmm}$ space group, 77 hosting a typical Kagome structure composed of vanadium Kagome net (Fig. 1a). In the 78 CDW phase, a distortion of the V-Kagome lattice engenders the $2 \times 2$ reconstruction and 79 forms a tri-hexagonal $(\operatorname{TrH})$ structure on the V-Kagome plane (Fig. 1b)[15, 25]. Such a so lattice distortion leads to Brillouin zone reconstruction in the reciprocal space which can be 81 described by three wavevectors (Fig. 1d). 
${ }_{82}$ Figure 1e shows the Fermi surface mapping of $\mathrm{KV}_{3} \mathrm{Sb}_{5}$ measured at $20 \mathrm{~K}$ in the $\mathrm{CDW}$ 83 state. Extended momentum space that includes both the first and second Brillouin zones 84 is covered in our measurements. This is important to obtain complete Fermi surface since ${ }_{85}$ the band structures of $\mathrm{KV}_{3} \mathrm{Sb}_{5}$ exhibit significant photoemission matrix element effects in 86 different momentum space (Fig. 1e, Fig. S2 and S3). The Fermi surface mapping in Fig. ${ }_{87} 1$ e, combined with the analysis of the related constant energy contours (Fig. S2) and band 88 structures (Fig. S3), gives rise to a Fermi surface topology that is mainly composed of a s9 circular electron-like pocket around $\bar{\Gamma}(\alpha)$, a large hexagon-shaped hole-like sheet centered 9o around $\bar{\Gamma}(\beta)$, a triangular hole-like pocket around $\bar{K}(\gamma)$ and a triangular electron-like ${ }_{91}$ pocket around $\bar{K}(\delta)$ as marked in Fig. 1e. The $\gamma$ pocket is clearly visualized around $\bar{K}_{21}$ 92 but is weak around $\bar{K}_{12}$; its size increases with increasing binding energy in the measured ${ }_{93}$ constant energy contours (Fig. S2). On the other hand, the $\delta$ pocket is clearly observed ${ }_{94}$ around $\bar{K}_{12}$ but is weak around $\bar{K}_{21}$; its size decreases with increasing binding energy in the ${ }_{95}$ constant energy contours (Fig. S2). The quantitatively extracted Fermi surface is shown ${ }_{96}$ in Fig. 1f, which agree well with the calculated Fermi surface of $\mathrm{KV}_{3} \mathrm{Sb}_{5}$ at $k_{z}=0.5$ in its ${ }_{97}$ pristine structure in Fig. 1g.

98 The CDW-related $2 \times 2$ lattice reconstruction is expected to generate electronic structure 99 reconstruction, as illustrated in Fig. 1d. However, no signature of such electronic reconstruc100 tion has been detected in the previous ARPES measurements[2, 37-44]. We have observed ${ }_{101}$ clear evidence of electronic structure reconstruction induced by the $2 \times 2 \mathrm{CDW}$ transition in ${ }_{102} \mathrm{KV}_{3} \mathrm{Sb}_{5}$ both in the measured Fermi surface and the band structure. Fig. 2a replots the ${ }_{103}$ Fermi surface mapping of $\mathrm{KV}_{3} \mathrm{Sb}_{5}$ shown in Fig. 1e, focusing on the first Brillouin zone. In 104 addition to the main Fermi surface, some additional features are clearly observed, as marked 105 by the arrows in Fig. 2a. Fig. $2 b$ shows the effect of the $2 \times 2$ lattice reconstruction on the ${ }_{106}$ Fermi surface as induced by one of the three wavevectors, $Q_{1}$. The reconstructed Fermi ${ }_{107}$ surface sheets (dashed lines in Fig. 2b) are produced from shifting the original $\alpha, \beta, \gamma$ and $\delta$ 108 main Fermi surface (solid lines in Fig. 2b) by the wavevector of $\pm Q_{1}$. As shown in Fig. 2a, 109 the extra features can be attributed to the reconstructed Fermi surface because the observed 110 features $(1,2),(3,4)$ and 5 agree well with the reconstructed $\delta, \beta$, and $\alpha$ Fermi surface, 111 respectively. The electronic reconstruction is also directly evidenced in the measured band ${ }_{112}$ structure in Fig. 2c, in which the band measured along the $\bar{\Gamma}-\bar{M}$ direction coincides with 113 the direction of $Q_{1}$ wavevector. As shown in Fig. 2c, in addition to the main $\beta$ bands, some 
114 extra bands are clearly observed around $\bar{\Gamma}\left(\beta_{L}^{\prime}\right.$ and $\left.\beta_{R}^{\prime}\right)$. The extra feature around $\bar{\Gamma}$ resem115 bles the strong $\beta$ band at $\bar{M}$. Quantitative analysis of the momentum distribution curve 116 (MDC) at the Fermi level in Fig. 2d indicates that the two extra features at $\bar{\Gamma}\left(\beta_{L}^{\prime}\right.$ and $\left.\beta_{R}^{\prime}\right)$ ${ }_{117}$ are separated from the $\beta$ band at $\bar{M}\left(\beta_{L}\right.$ and $\left.\beta_{R}\right)$ by exactly a wavevector of $Q_{1}$. Further 118 analysis of the photoemission spectra (energy distribution curves, EDCs) at $\bar{M}$ and $\bar{\Gamma}$ in Fig. ${ }_{119} 2 \mathrm{e}$ indicates that they have similar lineshape near the Fermi level within an energy range of ${ }_{120} \sim 0.3 \mathrm{eV}$. These results strongly demonstrate that the extra features at $\bar{\Gamma}$ are replicas of the ${ }_{121} \beta$ band at $\bar{M}$ caused by the $2 \times 2 \mathrm{CDW}$ modulation.

122 Besides the electronic structure reconstruction, the manifestations of the CDW transition ${ }_{123}$ involve the opening of the CDW gap, both at the Fermi level and away from the Fermi 124 level. We have clearly observed the CDW gap openings for both cases. Fig. 3 shows band ${ }_{125}$ structures of $\mathrm{KV}_{3} \mathrm{Sb}_{5}$ measured along high-symmetry directions $\bar{\Gamma}-\bar{M}$ (Fig. 3a and 3d), $\bar{K}$ ${ }_{126} \bar{M}-\bar{K}$ (Fig. 3b and 3e) and $\bar{\Gamma}-\bar{K}$ (Fig. 3c and 3f) at $20 \mathrm{~K}$. For comparison, we also present ${ }_{127}$ the calculated band structures for both pristine (Fig. 3g) and reconstructed (Fig. 3h) crystal 128 structures. In the calculated band strcture for the pristine lattice structure (Fig. 3g), the 129 bands around the Fermi level originate mainly from the $5 p$ orbitals of $\mathrm{Sb}(\alpha$ band from the ${ }_{130}$ in-plane $\mathrm{Sb}$ while $\gamma_{2}$ band from the out-of-plane $\left.\mathrm{Sb}\right)$ and the $3 d$ orbitals of $\mathrm{V}\left(\beta, \gamma_{1}\right.$ and $\delta$ 131 bands). The $\delta$ band originates from the V-Kagome lattice with the prototypical Dirac point 132 at $\bar{K}$ and von Hove singularities at $\bar{M}$. The $\beta$ band also comes from V-Kagome lattice with 133 different orbital character. The $2 \times 2$ lattice reconstruction causes significant modifications 134 of the band structures, manifested mainly by the band splitting and the associated CDW ${ }_{135}$ gap opening at $\bar{M}$ in the original Brillouin zone and $\bar{M}^{\prime}$ in the reconstructed Brillouin zone. 136 As shown in the calculated band structure for the reconstructed lattice in Fig. 3h, within ${ }_{137}$ the energy of interest, three CDW gaps open at $\bar{M}: \bar{M} \mathrm{G} 1$ from $\delta_{1}$ band, $\bar{M} \mathrm{G} 2$ from $\zeta$ band ${ }_{138}$ and $\bar{M} \mathrm{G} 3$ from $\delta_{2}$ band. In the meantime, four CDW gaps open at $\bar{M}^{\prime}$ point: $\bar{M}$ PG1 from ${ }_{139} \delta_{2}$ band, $\bar{M}$ PG2 from $\gamma_{1}$ band, $\bar{M}$ PG3 from $\beta_{2}$ band and $\bar{M}$ PG4 from $\delta_{1}$ band. In addition, 140 the spin-orbit coupling (SOC) is expected to open a gap at the Dirac point formed from the ${ }_{141} \delta$ bands at $\bar{K}$, as marked by DG in Fig. $3 g$ and $3 \mathrm{~h}$.

${ }_{142}$ The expected band splittings and CDW gap openings at $\bar{M}$ and $\bar{M}^{\prime}$ below the Fermi ${ }_{143}$ level are clearly observed in the measured band structures of $\mathrm{KV}_{3} \mathrm{Sb}_{5}$. Fig. 3d and 3e show 144 the CDW gap openings at the $\bar{M}$ point where the $\zeta$ band opens a gap labeled as $\bar{M}$ G2 145 and the $\delta_{2}$ band opens a gap $\bar{M} \mathrm{G} 3$. In the corresponding EDCs at $\bar{M}$, signatures of these 
${ }_{146}$ two gap openings can also be clearly visualized with the gap size of $\sim 150 \mathrm{meV}$ for $\bar{M} \mathrm{G} 2$ 147 and $\sim 125 \mathrm{meV}$ for $\bar{M} \mathrm{G} 3$. Fig. 3f shows the CDW gap openings at the $\bar{M}^{\prime}$ point where ${ }_{148}$ the $\beta_{2}$ band opens a gap labeled as $\bar{M}$ PG3 and the $\delta_{1}$ band opens a gap $\bar{M}$ PG4. In the 149 corresponding EDC at $\bar{M}^{\prime}$ in Fig. 3j, the $\bar{M}$ PG4 gap can be clearly determined with a gap 150 size of $\sim 150 \mathrm{meV}$. The $\bar{M}$ PG3 gap is present as seen from the dip in EDC near the binding 151 energy of $300 \mathrm{meV}$ that corresponds to the spectral weight suppression in the region pointed 152 out by the arrow in Fig. 3c. However, the related band is weak; its gap size is hard to be 153 determined precisely but estimated to be $\sim 150 \mathrm{meV}$. The SOC gap opening of the Dirac ${ }_{154}$ point at $\bar{K}$ can be seen from the EDCs in Fig. 3k; the measured gap size is $\sim 80 \mathrm{meV}$. 155 The measured band splittings and gap openings agree well with those from band structure 156 calculations.

157 Now we come to the CDW gap on the Fermi surface. To this end, we took high energy158 resolution $(\sim 4 \mathrm{meV})$ ARPES measurements on $\mathrm{KV}_{3} \mathrm{Sb}_{5}$ at $5 \mathrm{~K}$, covering the momentum 159 space around $\bar{M}_{21}$ as shown in Fig. 4l. In this region, in addition to the well-resolved $\alpha$ 160 and $\beta$ Fermi surface sheets, the $\gamma$ and $\delta$ sheets are also well separated because the former 161 is strong around $\bar{K}_{21}$ while the latter is strong around $\bar{K}_{12}$. The clearly distinguished four ${ }_{162}$ Fermi surface sheets facilitate the extraction of the Fermi surface-dependent and momentum${ }_{163}$ dependent CDW gaps. Fig. 4a-e show the symmetrized EDCs along the four Fermi surface; ${ }_{164}$ the data are taken on the two $\beta$ sheets on the two sides of $\bar{M}_{21}$ for confirming the data 165 reliability. In the symmetrized EDCs, the gap opening causes a spectral weight suppression 166 near the Fermi level that gives rise to a dip at the Fermi level; the gap size can be determined 167 by the peak position near the Fermi level. The extracted CDW gaps along the four Fermi 168 surface sheets are plotted in Fig. 4f-j. No CDW gap opening is observed around the $\alpha$ ${ }_{169}$ Fermi surface as shown in Fig. $4 \mathrm{a}$ and $4 \mathrm{f}$. For the $\beta$ Fermi surface, both measurements 170 in Fig. 4b and 4c give a consistent result on the CDW gap in Fig. 4g and 4h. The ${ }_{171}$ CDW gap on the $\beta$ Fermi surface is anisotropic; it shows a minimum close to zero along 172 the $\bar{\Gamma}-\bar{M}$ and $\bar{\Gamma}-\bar{K}$ directions but exhibits a maximum in the middle between these two ${ }_{173}$ directions. The CDW gaps along the $\gamma$ and $\delta$ Fermi surface sheets show similar behaviors, 174 as seen in Fig. 4(d,e) and Fig. 4(i,j). They are both anisotropic, showing a minimum 175 along the $\bar{\Gamma}-\bar{K}$ direction and a maximum along the $\bar{K}-\bar{M}$ direction. The EDCs along the $\gamma$ 176 and $\delta$ Fermi surface also show multiple features (Fig. 4d and 4e); besides the low energy 177 peak, there is another peak at a higher binding energy around $70 \mathrm{meV}$. As we will show 
178 below, such a peak-dip-hump structure can be attributed to the electron-phonon coupling. ${ }_{179}$ Fig. 4k shows a three-dimensional picture summarizing the Fermi surface-dependent and 180 momentum-dependent CDW gaps we have observed in $\mathrm{KV}_{3} \mathrm{Sb}_{5}$.

181 The CDW transition usually involves electronic structure reconstruction and lattice dis182 tortion in which the electron-phonon coupling plays an important role[45]. We have obtained 183 clear evidence of electron-phonon coupling in $\mathrm{KV}_{3} \mathrm{Sb}_{5}$. Fig. $5 \mathrm{a}-5 \mathrm{c}$ zoom in on the band struc184 tures of $\mathrm{KV}_{3} \mathrm{Sb}_{5}$ near the Fermi level measured along $\bar{\Gamma}-\bar{K}, \bar{K}-\bar{M}-\bar{K}$ and $\bar{\Gamma}-\bar{M}-\bar{\Gamma}$ directions 185 at $20 \mathrm{~K}$ in the $\mathrm{CDW}$ state. The corresponding EDCs are shown in Fig. 5d-5f. For the $\delta$ 186 band in Fig. 5a, $\gamma$ and $\delta$ bands in Fig. $5 \mathrm{~b}$ and $\beta$ band in Fig. 5c, the peak-dip-hump ${ }_{187}$ structure is clearly observed near their respective Fermi momenta as the peaks are marked 188 by triangles and the humps are marked by bars in Fig. 5d-5f. Fig. 5g shows the expanded 189 view of the $\delta$ band in Fig. 5a. A kink in the dispersion can be observed as marked by 190 arrow in Fig. 5g. The quantitative dispersion is obtained by fitting momentum distribution 191 curves (MDCs) at different binding energies and plotted on top of the observed band in Fig. ${ }_{192} 5 \mathrm{~g}$. Taking a linear line as an empirical bare band, the effective real part of the electron ${ }_{193}$ self-energy is shown in Fig. $5 \mathrm{~h}$. It shows a clear peak at $\sim 36 \mathrm{meV}$. The observed kink in the 194 energy dispersion and the peak-dip-hump structure in EDCs are reminiscent of those from 195 the electron-boson coupling in simple metal[46] and high-temperature superconductors[47]. 196 The phonon frequency of the vanadium vibrations in $\mathrm{AV}_{3} \mathrm{Sb}_{5}$ can reach up to $\sim 36 \mathrm{meV}$ [32] ${ }_{197}$ that is consistent with the mode energy we have observed. Therefore, we have observed ${ }_{198}$ signatures of the electron-phonon coupling in $\mathrm{KV}_{3} \mathrm{Sb}_{5}$ and such electron-phonon coupling is 199 present for all the $\beta, \gamma$ and $\delta$ bands.

200 The CDW state is first proposed for a one-dimensional chain of atoms with an equal spac201 ing $a$ which is argued to be inherently unstable against the dimerized ground state[48]. This 202 would open a CDW gap at the Fermi point $k_{F}= \pm \pi / 2 a$ and produce a lattice reconstruction 203 with a wavevector of $\pi / a$. Such a Fermi surface nesting picture is extended to real materials 204 with higher dimensions where the CDW state is realized because segments of the Fermi 205 surface are connected by a wavevector $Q_{C D W}[45]$. This would give rise to a partial CDW 206 gap opening on the Fermi surface and reconstructions of both the electronic structure and ${ }_{207}$ the lattice with a wavevector of $Q_{C D W}$. Besides the Fermi surface nesting, the CDW phase ${ }_{208}$ can also be driven by the concerted action of electronic and ionic subsystems where a q${ }_{209}$ dependent electron-phonon coupling plays an indispensable part [49, 50]. In $\mathrm{AV}_{3} \mathrm{Sb}_{5}$ system, 
${ }_{210}$ the driving force for the CDW formation remains under debate[15, 17, 25, 32, 33, 37, 38]. ${ }_{211}$ Based on our observations, we found that the electron-phonon coupling plays a major role ${ }_{212}$ in generating the $\mathrm{CDW}$ phase in $\mathrm{KV}_{3} \mathrm{Sb}_{5}$. Firstly, the measured Fermi surface (Fig. 1f) ${ }_{213}$ and band structures (Fig. 3a-3c) of $\mathrm{KV}_{3} \mathrm{Sb}_{5}$ show a high agreement with the band structure ${ }_{214}$ calculations that do not incorporate the electron-electron interactions, which indicates the 215 electron correlation effect is weak in $\mathrm{KV}_{3} \mathrm{Sb}_{5}$. Secondly, besides the gap opening around the ${ }_{216}$ Fermi sueface, we have also observed clear CDW gap opening at $\bar{M}$ and $\bar{M}^{\prime}$ with a gap size ${ }_{217}$ up to $\sim 150 \mathrm{meV}$ (Fig. 3) highly away from the Fermi level. Thirdly, the electron-phonon ${ }_{218}$ couplings on the $\beta, \gamma$ and $\delta$ bands are directly observed (Fig. 5). All these results indicate ${ }_{219}$ that the $\mathrm{CDW}$ phase in $\mathrm{KV}_{3} \mathrm{Sb}_{5}$ is mainly driven by the electron-phonon coupling induced 220 structural phase transition.

${ }_{221}$ In summary, through our high-resolution ARPES measurements and the density func222 tional theory (DFT) calculations on $\mathrm{KV}_{3} \mathrm{Sb}_{5}$, clear evidence of the $2 \times 2 \mathrm{CDW}$-induced elec223 tronic structure reconstruction has been uncovered. These include the Fermi surface re224 construction, the associated band structure foldings, and the CDW gap openings at the 225 boundary of the pristine and reconstructed Brillouin zone. The Fermi surface-dependent 226 and momentum-dependent CDW gap is measured for the first time and strong anisotropy of ${ }_{227}$ the CDW gap is observed for all the V-derived Fermi surface sheets. The electron-phonon 228 couplings have been observed for all the V-derived bands. These results indicate that the 229 electron correlation effect in $\mathrm{KV}_{3} \mathrm{Sb}_{5}$ is weak and the electron-phonon coupling plays a dom230 inant role in driving the CDW transition. They provide key information in understanding ${ }_{231}$ the origin of the $\mathrm{CDW}$ state and its interplay with superconductivity in $\mathrm{AV}_{3} \mathrm{Sb}_{5}$ supercon232 ductors.

\section{${ }_{233}$ Methods}

${ }_{234}$ Growth and characterization of single crystals. High quality single crystals of ${ }_{235} \mathrm{KV}_{3} \mathrm{Sb}_{5}$ were grown from a two-steps flux method[1]. First, $\mathrm{KSb}_{2}$ alloy was sintered at ${ }_{236} 573 \mathrm{~K}$ for 20 hours in an alumina crucible coated with aluminum foil. Second, high-purity ${ }_{237} \mathrm{~K}, \mathrm{~V}, \mathrm{Sb}$ and $\mathrm{KSb}_{2}$ precursor were mixed in a molar ratio of 1:3:14:10 and then sealed in a Ta 238 tube. The tube was sealed in an evacuated quartz ampoule, heated up to $1273 \mathrm{~K}$, soaked for ${ }_{239} 20$ hours and then cooled down to $773 \mathrm{~K}$ at a rate of $2 \mathrm{~K} /$ hour. Shiny lamellar crystals were 240 separated from the flux by centrifuging with a regular hexagon shape and a size up to $4 \times 4$ ${ }_{241} \mathrm{~mm}^{2}$ (inset of Fig. S1a in Supplementary Materials). The crystals were characterized by 
${ }_{242} \mathrm{X}$-ray diffraction (Fig. S1a) and their magnetic susceptibilty and resistance were measured ${ }_{243}$ (Fig. S1b and S1c). The CDW transition temperature, $T_{C D W}$, is $\sim 80 \mathrm{~K}$ from the magnetic 244 measurement in Fig. S1b.

245 High resolution ARPES measurements High-resolution angle-resolved photoemis246 sion measurements were carried out on our lab system equipped with a Scienta R4000 elec${ }_{247}$ tron energy analyzer[51]. We use helium discharge lamp as the light source that can provide 248 a photon energy of $h \nu=21.218 \mathrm{eV}$ (helium I). The energy resolution was set at $\sim 20 \mathrm{meV}$ ${ }_{249}$ for the Fermi-surface mapping (Fig. 1) and band-structure (Fig. 2, 3 and 5) measurements 250 and at $4 \mathrm{meV}$ for the CDW gap measurements (Fig. 4). The angular resolution is $\sim 0.3^{\circ}$. ${ }_{251}$ The Fermi level is referenced by measuring on a clean polycrystalline gold that is electrically ${ }_{252}$ connected to the sample. The sample was cleaved in situ and measured in vacuum with a 253 base pressure better than $1.2 \times 10^{-10}$ Torr.

${ }^{254}$ Calculations. First-principles calculations are performed by using the Projected Aug255 mented Wave Method (PAW) within the spin-polarized density functional theory (DFT), 256 as implemented in the Vienna Ab Initio Simulation Package (VASP)[52-54]. We construct ${ }_{257} 2 \times 2 \times 1$ supercell to describe the $\mathrm{TrH} \mathrm{CDW}$ phase of $\mathrm{KV}_{3} \mathrm{Sb}_{5}$. The crystal structures are ${ }_{258}$ relaxed by using the Perdew-Burke-Ernzerhof (PBE) functional[55] and zero damping DFT${ }_{259} \mathrm{D} 3$ van der Walls correction[56] until the forces are less than $0.001 \mathrm{eV} / \AA$. The cutoff energy 260 of plane wave basis is set as $600 \mathrm{eV}$ and the energy convergence criterion is set as $10^{-7} \mathrm{eV}$. ${ }_{261}$ The corresponding Brillouin zones are sampled by using a $16 \times 16 \times 10$ (for primitive cell) and 262 a $8 \times 8 \times 10$ (for supercell) Gamma centered k-grid. The effective band structure is calculated 263 by the band-unfolding method[57, 58] proposed by Zunger et al. with BandUP code[59, 60].

264

${ }_{265}$ Acknowledgement

${ }_{266}$ This work is supported by the National Natural Science Foundation of China (Grant ${ }_{267}$ Nos. 11888101, 11922414, 11974404, 12074411 and U2032204), the National Key Research 268 and Development Program of China (Grant Nos. 2016YFA0300300, 2016YFA0300602, 269 2017YFA0302900, 2017YFA0303100, 2018YFA0305602 and 2018YFA0704200), the Strategic ${ }_{270}$ Priority Research Program (B) of the Chinese Academy of Sciences (Grant No. XDB25000000, ${ }_{271}$ XDB28000000 and XDB33000000), the Youth Innovation Promotion Association of CAS ${ }_{272}$ (Grant No. 2017013), the Research Program of Beijing Academy of Quantum Information 
${ }_{273}$ Sciences (Grant No. Y18G06) and the K. C. Wong Education Foundation (GJTD-2018-01). ${ }_{274}$ Author Contributions

${ }_{275}$ X.J.Z. and H.L.L. conceived this project. H.L.L. and Q.G. performed ARPES experiments. ${ }_{276}$ H.L.L. and Q.G. analyze the ARPES data. H.X.L. , C.J.Y. and Y.G.S. contributed to crystal 277 growth. Y.H.G., K.J. and J.P.H. contributed to DFT calculations. D.S.W., J.J.J., S.L.W., ${ }_{278}$ X.Y.L., Y.X., L.Z., Q.Y.W., H.Q.M., G.D.L., Z.H.Z., Z.Y.X. and X.J.Z. contributed to the 279 development and maintenance of the ARPES systems and related software development. 280 H.L.L., K.J. and X.J.Z. wrote this paper. All authors participated in discussion and comment 281 on the paper.

[1] Brenden R. Ortiz, Lidia C. Gomes, Jennifer R. Morey, Michal Winiarski, Mitchell Bordelon, John S. Mangum, Iain W. H. Oswald, Jose A. Rodriguez-Rivera, James R. Neilson, Stephen D. Wilson, Elif Ertekin, Tyrel M. McQueen, and Eric S. Toberer. New kagome prototype materials: discovery of $\mathrm{KV}_{3} \mathrm{Sb}_{5}, \mathrm{RbV}_{3} \mathrm{Sb}_{5}$, and $\mathrm{CsV}_{3} \mathrm{Sb}_{5}$. Physical Review Materials, 3(9):094407, 2019 .

[2] Brenden R. Ortiz, Samuel M. L. Teicher, Yong Hu, Julia L. Zuo, Paul M. Sarte, Emily C. Schueller, A. M. Milinda Abeykoon, Matthew J. Krogstad, Stephan Rosenkranz, Raymond Osborn, Ram Seshadri, Leon Balents, Junfeng He, and Stephen D. Wilson. $\mathrm{CsV}_{3} \mathrm{Sb}_{5}$ : a $\mathrm{Z}_{2}$ topological kagome metal with a superconducting ground state. Physical Review Letters, 125(24):247002, 2020.

[3] H. M. Guo and M. Franz. Topological insulator on the kagome lattice. Physical Review B, 80(11):113102, 2009.

[4] A. O'Brien, F. Pollmann, and P. Fulde. Strongly correlated fermions on a kagome lattice. Physical Review B, 81(23):235115, 2010.

[5] Hao Yang, Yan Sun, Yang Zhang, Wu-Jun Shi, Stuart S. P. Parkin, and Binghai Yan. Topological Weyl semimetals in the chiral antiferromagnetic materials $\mathrm{Mn}_{3} \mathrm{Ge}$ and $\mathrm{Mn}_{3} \mathrm{Sn}$. New Journal of Physics, 19(1):015008, 2017.

[6] Andreas Rüegg and Gregory A. Fiete. Fractionally charged topological point defects on the kagome lattice. Physical Review B, 83(16):165118, 2011.

[7] S. V. Isakov, S. Wessel, R. G. Melko, K. Sengupta, and Yong Baek Kim. Hard-Core bosons on 
the kagome lattice: valence-bond solids and their quantum melting. Physical Review Letters, 97(14):147202, 2006.

[8] Wan-Sheng Wang, Zheng-Zhao Li, Yuan-Yuan Xiang, and Qiang-Hua Wang. Competing electronic orders on kagome lattices at van Hove filling. Physical Review B, 87(11):115135, 2013.

[9] Wing-Ho Ko, Patrick A. Lee, and Xiao-Gang Wen. Doped kagome system as exotic superconductor. Physical Review B, 79(21):214502, 2009.

[10] Maximilian L. Kiesel and Ronny Thomale. Sublattice interference in the kagome Hubbard model. Physical Review B, 86(12):121105, 2012.

[11] Maximilian L. Kiesel, Christian Platt, and Ronny Thomale. Unconventional Fermi surface instabilities in the kagome Hubbard model. Physical Review Letters, 110(12):126405, 2013.

[12] Shuo-Ying Yang, Yaojia Wang, Brenden R. Ortiz, Defa Liu, Jacob Gayles, Elena Derunova, Rafael Gonzalez-Hernandez, Libor Šmejkal, Yulin Chen, Stuart S. P. Parkin, Stephen D. Wilson, Eric S. Toberer, Tyrel McQueen, and Mazhar N. Ali. Giant, unconventional anomalous Hall effect in the metallic frustrated magnet candidate, $\mathrm{KV}_{3} \mathrm{Sb}_{5}$. Science Advances, 6(31):eabb6003, 2020.

[13] F. H. Yu, T. Wu, Z. Y. Wang, B. Lei, W. Z. Zhuo, J. J. Ying, and X. H. Chen. Concurrence of anomalous Hall effect and charge density wave in a superconducting topological kagome metal. arXiv:2102.10987, 2021.

[14] Eric M. Kenney, Brenden R. Ortiz, Chennan Wang, Stephen D. Wilson, and Michael J. Graf. Absence of local moments in the kagome metal $\mathrm{KV}_{3} \mathrm{Sb}_{5}$ as determined by muon spin spectroscopy. Journal of Physics: Condensed Matter, 33(23):235801, 2021.

[15] Yu-Xiao Jiang, Jia-Xin Yin, M. Michael Denner, Nana Shumiya, Brenden R. Ortiz, Gang Xu, Zurab Guguchia, Junyi He, Md Shafayat Hossain, Xiaoxiong Liu, Jacob Ruff, Linus Kautzsch, Songtian S. Zhang, Guoqing Chang, Ilya Belopolski, Qi Zhang, Tyler A. Cochran, Daniel Multer, Maksim Litskevich, Zi-Jia Cheng, Xian P. Yang, Ziqiang Wang, Ronny Thomale, Titus Neupert, Stephen D. Wilson, and M. Zahid Hasan. Discovery of unconventional chiral charge order in kagome superconductor $\mathrm{KV}_{3} \mathrm{Sb}_{5}$. arXiv:2012.15709, 2020.

[16] Hui Chen, Haitao Yang, Bin Hu, Zhen Zhao, Jie Yuan, Yuqing Xing, Guojian Qian, Zihao Huang, Geng Li, Yuhan Ye, Qiangwei Yin, Chunsheng Gong, Zhijun Tu, Hechang Lei, Shen Ma, Hua Zhang, Shunli Ni, Hengxin Tan, Chengmin Shen, Xiaoli Dong, Binghai Yan, Ziqiang 
Wang, and Hong-Jun Gao. Roton pair density wave and unconventional strong-coupling superconductivity in a topological kagome metal. arXiv:2103.09188, 2021.

[17] H. X. Li, T. T. Zhang, Y. Y. Pai, C. Marvinney, A. Said, T. Yilmaz, Q. Yin, C. Gong, Z. Tu, E. Vescovo, R. G. Moore, S. Murakami, H. C. Lei, H. N. Lee, B. Lawrie, and H. Miao. Observation of unconventional charge density wave without acoustic phonon anomaly in kagome Superconductors $\mathrm{AV}_{3} \mathrm{Sb}_{5}(\mathrm{~A}=\mathrm{Rb}, \mathrm{Cs})$. arXiv:2103.09769, 2021.

[18] C. C. Zhao, L. S. Wang, W. Xia, Q. W. Yin, J. M. Ni, Y. Y. Huang, C. P. Tu, Z. C. Tao, Z. J. Tu, C. S. Gong, H. C. Lei, Y. F. Guo, X. F. Yang, and S. Y. Li. Nodal superconductivity and superconducting domes in the topological kagome metal $\mathrm{CsV}_{3} \mathrm{Sb}_{5}$. arXiv:2102.08356, 2021.

[19] Zuowei Liang, Xingyuan Hou, Fan Zhang, Wanru Ma, Ping Wu, Zongyuan Zhang, Fanghang Yu, J. J. Ying, Kun Jiang, Lei Shan, Zhenyu Wang, and X. H. Chen. Three-dimensional charge density wave and robust zero-bias conductance peak inside the superconducting vortex core of a kagome superconductor $\mathrm{CsV}_{3} \mathrm{Sb}_{5}$. arXiv:2103.04760, 2021.

[20] Weiyin Duan, Zhiyong Nie, Shuaishuai Luo, Fanghang Yu, Brenden R. Ortiz, Lichang Yin, Hang Su, Feng Du, An Wang, Ye Chen, Xin Lu, Jianjun Ying, Stephen D. Wilson, Xianhui Chen, Yu Song, and Huiqiu Yuan. Nodeless superconductivity in the kagome metal $\mathrm{CsV}_{3} \mathrm{Sb}_{5}$. arXiv:2103.11796, 2021.

[21] Chao Mu, Qiangwei Yin, Zhijun Tu, Chunsheng Gong, Hechang Lei, Zheng Li, and Jianlin Luo. $s$-wave superconductivity in kagome metal $\mathrm{CsV}_{3} \mathrm{Sb}_{5}$ revealed by ${ }^{121 / 123} \mathrm{Sb} \mathrm{NQR}$ and ${ }^{51} \mathrm{~V}$ NMR measurements. arXiv:2104.06698, 2021.

[22] Han-Shu Xu, Ya-Jun Yan, Ruotong Yin, Wei Xia, Shijie Fang, Ziyuan Chen, Yuanji Li, Wenqi Yang, Yanfeng Guo, and Dong-Lai Feng. Multiband superconductivity with sign-preserving order parameter in kagome superconductor $\mathrm{CsV}_{3} \mathrm{Sb}_{5}$. arXiv:2104.08810, 2021.

[23] Brenden R. Ortiz, Paul M. Sarte, Eric M. Kenney, Michael J. Graf, Samuel M. L. Teicher, Ram Seshadri, and Stephen D. Wilson. Superconductivity in the $\mathrm{Z}_{2}$ kagome metal $\mathrm{KV}_{3} \mathrm{Sb}_{5}$. Physical Review Materials, 5(3):034801, 2021.

59 [24] Qiangwei Yin, Zhijun Tu, Chunsheng Gong, Yang Fu, Shaohua Yan, and Hechang Lei. Superconductivity and normal-state properties of kagome metal $\mathrm{RbV}_{3} \mathrm{Sb}_{5}$ single crystals. Chinese Physics Letters, 38(3):037403, 2021. 

390 [34] Xianxin Wu, Tilman Schwemmer, Tobias Müller, Armando Consiglio, Giorgio Sangiovanni,

density wave order in the kagome superconductor $\mathrm{CsV}_{3} \mathrm{Sb}_{5}$. arXiv:2104.07230, 2021.

26] K. Y. Chen, N. N. Wang, Q. W. Yin, Y. H. Gu, K. Jiang, Z. J. Tu, C. S. Gong, Y. Uwatoko, J. P. Sun, H. C. Lei, J. P. Hu, and J. G. Cheng. Double superconducting dome and triple enhancement of $T_{c}$ in the kagome superconductor $\mathrm{CsV}_{3} \mathrm{Sb}_{5}$ under high pressure. Physical Review Letters, 126(24):247001, 2021.

[27] Feng Du, Shuaishuai Luo, Brenden R. Ortiz, Ye Chen, Weiyin Duan, Dongting Zhang, Xin Lu, Stephen D. Wilson, Yu Song, and Huiqiu Yuan. Pressure-induced double superconducting domes and charge instability in the kagome metal $\mathrm{KV}_{3} \mathrm{Sb}_{5}$. Physical Review B, 103(22):L220504, 2021.

[28] F. H. Yu, D. H. Ma, W. Z. Zhuo, S. Q. Liu, X. K. Wen, B. Lei, J. J. Ying, and X. H. Chen. Unusual competition of superconductivity and charge-density-wave state in a compressed topological kagome metal. Nature Communications, 12(1):3645, 2021.

[29] Zhuyi Zhang, Zheng Chen, Ying Zhou, Yifang Yuan, Shuyang Wang, Jing Wang, Haiyang Yang, Chao An, Lili Zhang, Xiangde Zhu, Yonghui Zhou, Xuliang Chen, Jianhui Zhou, and Zhaorong Yang. Pressure-induced reemergence of superconductivity in the topological kagome metal $\mathrm{CsV}_{3} \mathrm{Sb}_{5}$. Physical Review B, 103(22):224513, 2021.

[30] Xu Chen, Xinhui Zhan, Xiaojun Wang, Jun Deng, Xiaobing Liu, Xin Chen, Jiangang Guo, and Xiaolong Chen. Highly robust reentrant superconductivity in $\mathrm{CsV}_{3} \mathrm{Sb}_{5}$ under pressure. Chinese Physical Letters, 38(5):057402, 2021.

[31] Alexander A. Tsirlin, Pierre Fertey, Brenden R. Ortiz, Berina Klis, Valentino Merkl, Martin Dressel, Stephen D. Wilson, and Ece Uykur. Anisotropic compression and role of Sb in the superconducting kagome metal $\mathrm{CsV}_{3} \mathrm{Sb}_{5}$. arXiv:2105.01397, 2021.

[32] Hengxin Tan, Yizhou Liu, Ziqiang Wang, and Binghai Yan. Charge density waves and electronic properties of superconducting kagome metals. arXiv:2103.06325, 2021.

[33] Xilin Feng, Kun Jiang, Ziqiang Wang, and Jiangping Hu. Chiral flux phase in the kagome superconductor $\mathrm{AV}_{3} \mathrm{Sb}_{5}$. arXiv:2103.07097, 2021.

Domenico Di Sante, Yasir Iqbal, Werner Hanke, Andreas P. Schnyder, M. Michael Denner, Mark H. Fischer, Titus Neupert, and Ronny Thomale. Nature of unconventional pairing in the kagome superconductors $\mathrm{AV}_{3} \mathrm{Sb}_{5}$. arXiv:2104.05671, 2021.

394 [35] Yu-Ping Lin and Rahul M. Nandkishore. Complex charge density waves at van Hove singularity 
423 [43] Soohyun Cho, Haiyang Ma, Wei Xia, Yichen Yang, Zhengtai Liu, Zhe Huang, Zhicheng Jiang,

on hexagonal lattices: Haldane-model phase diagram and potential realization in kagome metals $\mathrm{AV}_{3} \mathrm{Sb}_{5}$. arXiv:2104.02725, 2021.

36] H. Miao, H. X. Li, H. N. Lee, A. Said, H. C. Lei, J. X. Yin, M. Z. Hasan, Ziqiang Wang, Hengxin Tan, and Binghai Yan. Geometry of the charge density wave in kagome metal $\mathrm{AV}_{3} \mathrm{Sb}_{5}$. arXiv:2106.10150, 2021.

[37] Kosuke Nakayama, Yongkai Li, Min Liu, Zhiwei Wang, Takashi Takahashi, Yugui Yao, and Takafumi Sato. Multiple energy scales and anisotropic energy gap in the charge-density-wave phase of kagome superconductor $\mathrm{CsV}_{3} \mathrm{Sb}_{5}$. arXiv:2104.08042, 2021.

[38] Zhengguo Wang, Sheng Ma, Yuhang Zhang, Haitao Yang, Zhen Zhao, Yi Ou, Yu Zhu, Shunli Ni, Zouyouwei Lu, Hui Chen, Kun Jiang, Li Yu, Yan Zhang, Xiaoli Dong, Jiangping Hu, Hong-Jun Gao, and Zhongxian Zhao. Distinctive momentum dependent charge-density-wave gap observed in $\mathrm{CsV}_{3} \mathrm{Sb}_{5}$ superconductor with topological kagome lattice. arXiv:2104.05556, 2021.

[39] Yang Luo, Shuting Peng, Samuel M. L. Teicher, Linwei Huai, Yong Hu, Brenden R. Ortiz, Zhiyuan Wei, Jianchang Shen, Zhipeng Ou, Bingqian Wang, Yu Miao, Mingyao Guo, M. Shi, Stephen D. Wilson, and J. F. He. Distinct band reconstructions in kagome superconductor $\mathrm{CsV}_{3} \mathrm{Sb}_{5}$. arXiv:2106.01248, 2021.

Song, Zhengtai Liu, Dawei Shen, Yaobo Huang, Kai Liu, Hechang Lei, and Shancai Wang. Temperature-induced band renormalization and Lifshitz transition in a kagome superconductor $\mathrm{RbV}_{3} \mathrm{Sb}_{5}$. arXiv:2104.01125, 2021.

[41] Yong Hu, Samuel M. L. Teicher, Brenden R. Ortiz, Yang Luo, Shuting Peng, Linwei Huai, J. Z. Ma, N. C. Plumb, Stephen D. Wilson, J. F. He, and M. Shi. Charge-order-assisted topological surface states and flat bands in the kagome superconductor $\mathrm{CsV}_{3} \mathrm{Sb}_{5}$. arXiv:2104.12725, 2021.

[42] Mingu Kang, Shiang Fang, Jeong-Kyu Kim, Brenden R. Ortiz, Jonggyu Yoo, Byeong-Gyu Park, Stephen D. Wilson, Jae-Hoon Park, and Riccardo Comin. Twofold van Hove singularity and origin of charge order in topological kagome superconductor $\mathrm{CsV}_{3} \mathrm{Sb}_{5}$. arXiv:2105.01689, 2021.

${ }_{424}$ Xiangle Lu, Jishan Liu, Zhonghao Liu, Jinfeng Jia, Yanfeng Guo, Jianpeng Liu, and Dawei Shen. Emergence of new van Hove singularities in the charge density wave state of a topological 
${ }_{427}$ [44] Rui Lou, Alexander Fedorov, Qiangwei Yin, Andrii Kuibarov, Zhijun Tu, Chunsheng Gong,

431 [45] G. Grüner. The dynamics of charge-density waves. Reviews of Modern Physics, 60(4):1129$432 \quad 1181,1988$.

${ }_{433}$ [46] T. Valla, A. V. Fedorov, P. D. Johnson, and S. L. Hulbert. Many-body effects in angle-resolved

453 [53] P. E. Blöchl. Projector augmented-wave method. Physical Review B, 50(24):17953-17979,

$454 \quad 1994$.

455 [54] G. Kresse and J. Furthmüller. Efficient iterative schemes for ab initio total-energy calculations

456 using a plane-wave basis set. Physical Review B, 54(16):11169-11186, 1996. photoemission: quasiparticle energy and lifetime of a Mo(110) surface state. Physical Review Letters, 83(10):2085-2088, 1999.

47] A. Lanzara, P. V. Bogdanov, X. J. Zhou, S. A. Kellar, D. L. Feng, E. D. Lu, T. Yoshida, H. Eisaki, A. Fujimori, K. Kishio, J. I. Shimoyama, T. Noda, S. Uchida, Z. Hussain, and Z. X. Shen. Evidence for ubiquitous strong electron-phonon coupling in high-temperature superconductors. Nature, 412(6846):510-514, 2001.

48] R. E. Peierls. Quantum Theory of Solids. Oxford University, New York, 1955. in metals. Physical Review B, 77(16):165135, 2008.

[50] Xuetao Zhu, Yanwei Cao, Jiandi Zhang, E. W. Plummer, and Jiandong Guo. Classification of charge density waves based on their nature. Proceedings of the National Academy of Sciences, 112(8):2367, 2015.

[51] Guodong Liu, Guiling Wang, Yong Zhu, Hongbo Zhang, Guochun Zhang, Xiaoyang Wang, Yong Zhou, Wentao Zhang, Haiyun Liu, Lin Zhao, Jianqiao Meng, Xiaoli Dong, Chuangtian Chen, Zuyan $\mathrm{Xu}$, and $\mathrm{X}$. J. Zhou. Development of a vacuum ultraviolet laserbased angleresolved photoemission system with a superhigh energy resolution better than $1 \mathrm{meV}$. Review of Scientific Instruments, 79(2):023105, 2008.

[52] W. Kohn and L. J. Sham. Self-consistent equations including exchange and correlation effects. Physical Review, 140(4A):A1133-A1138, 1965. 
457 [55] John P. Perdew, Kieron Burke, and Matthias Ernzerhof. Generalized gradient approximation $458 \quad$ made simple. Physical Review Letters, 77(18):3865-3868, 1996.

459 [56] S. Grimme, J. Antony, S. Ehrlich, and H. Krieg. A consistent and accurate ab initio 460 parametrization of density functional dispersion correction (DFT-D) for the 94 elements H-Pu. $461 \quad$ J. Chem. Phys., 132(15):154104, 2010.

462 [57] Voicu Popescu and Alex Zunger. Effective band structure of random alloys. Physical Review $463 \quad$ Letters, 104(23):236403, 2010.

464 [58] Voicu Popescu and Alex Zunger. Extracting E versus k effective band structure from supercell 465 calculations on alloys and impurities. Physical Review B, 85(8):085201, 2012.

466 [59] Paulo VC Medeiros, Sven Stafström, and Jonas Björk. Effects of extrinsic and intrinsic per467 turbations on the electronic structure of graphene: Retaining an effective primitive cell band $468 \quad$ structure by band unfolding. Physical Review B, 89(4):041407, 2014.

469 [60] Paulo VC Medeiros, Stepan S Tsirkin, Sven Stafström, and Jonas Björk. Unfolding spinor 470 wave functions and expectation values of general operators: introducing the unfolding-density $471 \quad$ operator. Physical Review B, 91(4):041116, 2015. 

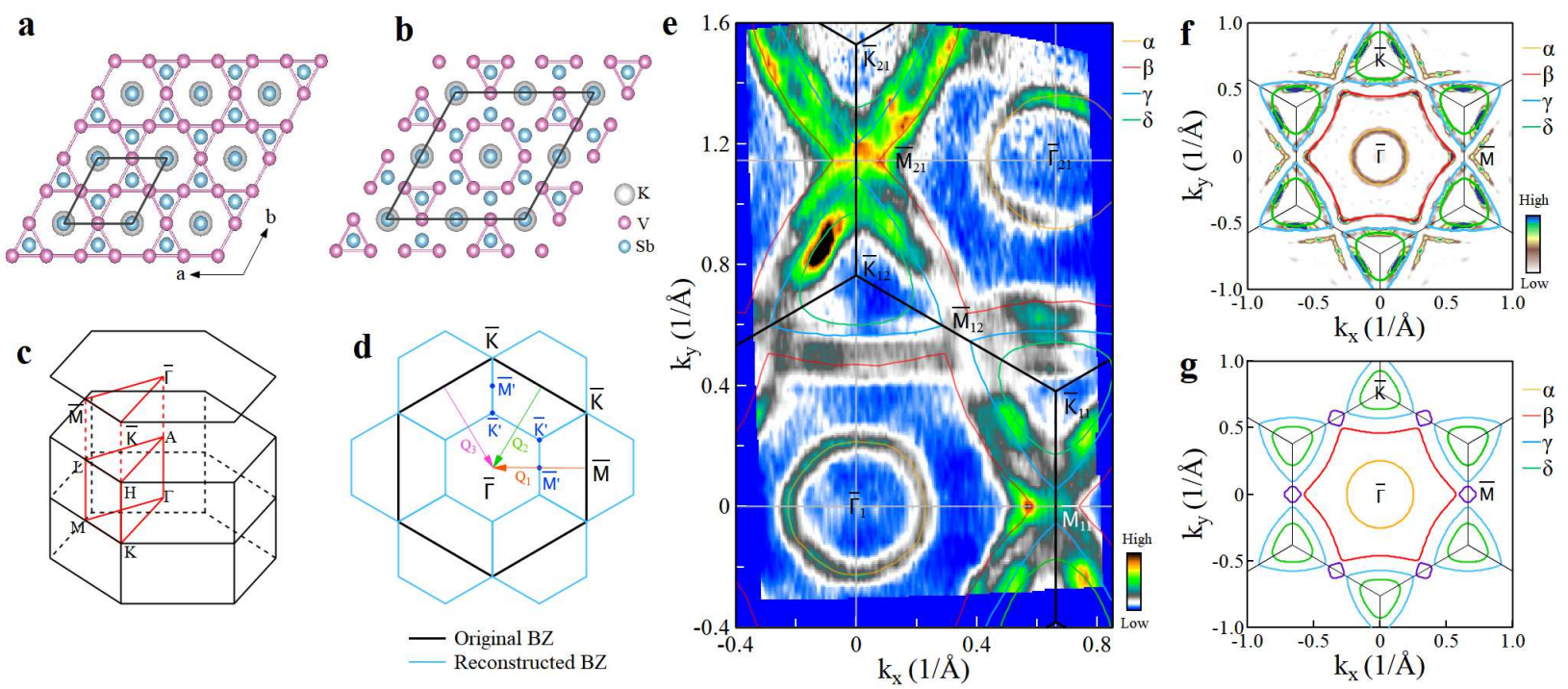

FIG. 1. Crystal structure and Fermi surface of $\mathbf{K V}_{3} \mathbf{S b}_{5}$. a, Pristine crystal strcture of $\mathrm{KV}_{3} \mathrm{Sb}_{5}$ with a V-kagome net from top view. b, The Tri-Hexagonal (TrH) lattice distortion caused by the $2 \times 2 \mathrm{CDW}$ transition[15, 25]. The K, V, Sb atoms are presented as grey, purple and blue balls, respectively. c, Schematic of the three-dimensional Brillouin zone and the two-dimentional Brillouin zone projected on the (001) surface in the pristine phase in (a). High-symmetry points and high-symmetry momentum lines are marked. d, The original (black lines) and $2 \times 2$ reconstructed (blue lines) Brillouin zones. The $\bar{\Gamma}, \bar{K}$ and $\bar{M}\left(\bar{\Gamma}^{\prime}, \bar{K}^{\prime}\right.$ and $\left.\bar{M}^{\prime}\right)$ are the high-symmetry points of the pristine $(2 \times 2$ reconstructed) Brillouin zones. The arrows indicate three wavevectors (marked as $Q_{1}, Q_{2}$ and $\left.Q_{3}\right)$ of electronic structure reconstruction. e, Fermi surface mapping of $\mathrm{KV}_{3} \mathrm{Sb}_{5}$ measured at $\mathrm{T}=20 \mathrm{~K}$. Four Fermi surface sheets are observed marked as $\alpha$ (orange lines), $\beta$ (red lines), $\gamma$ (blue lines) and $\delta$ (green lines). For convenience, high-symmetry points are labeled with different indexes such as $\bar{\Gamma}_{1}, \bar{\Gamma}_{21}, \bar{M}_{11}, \bar{M}_{12}, \bar{M}_{21}, \bar{K}_{11}, \bar{K}_{12}$ and $\bar{K}_{21}$. f, Symmetrized Fermi surface mapping of $\mathrm{KV}_{3} \mathrm{Sb}_{5}$. It is obtained from taking the second derivative with respect to momentum on the second Brillouin zone data in (e). $\mathrm{g}$, The calculated Fermi surface at $\mathrm{k}_{z}=0.5$ corresponding to pristine crystal structure in (a). 

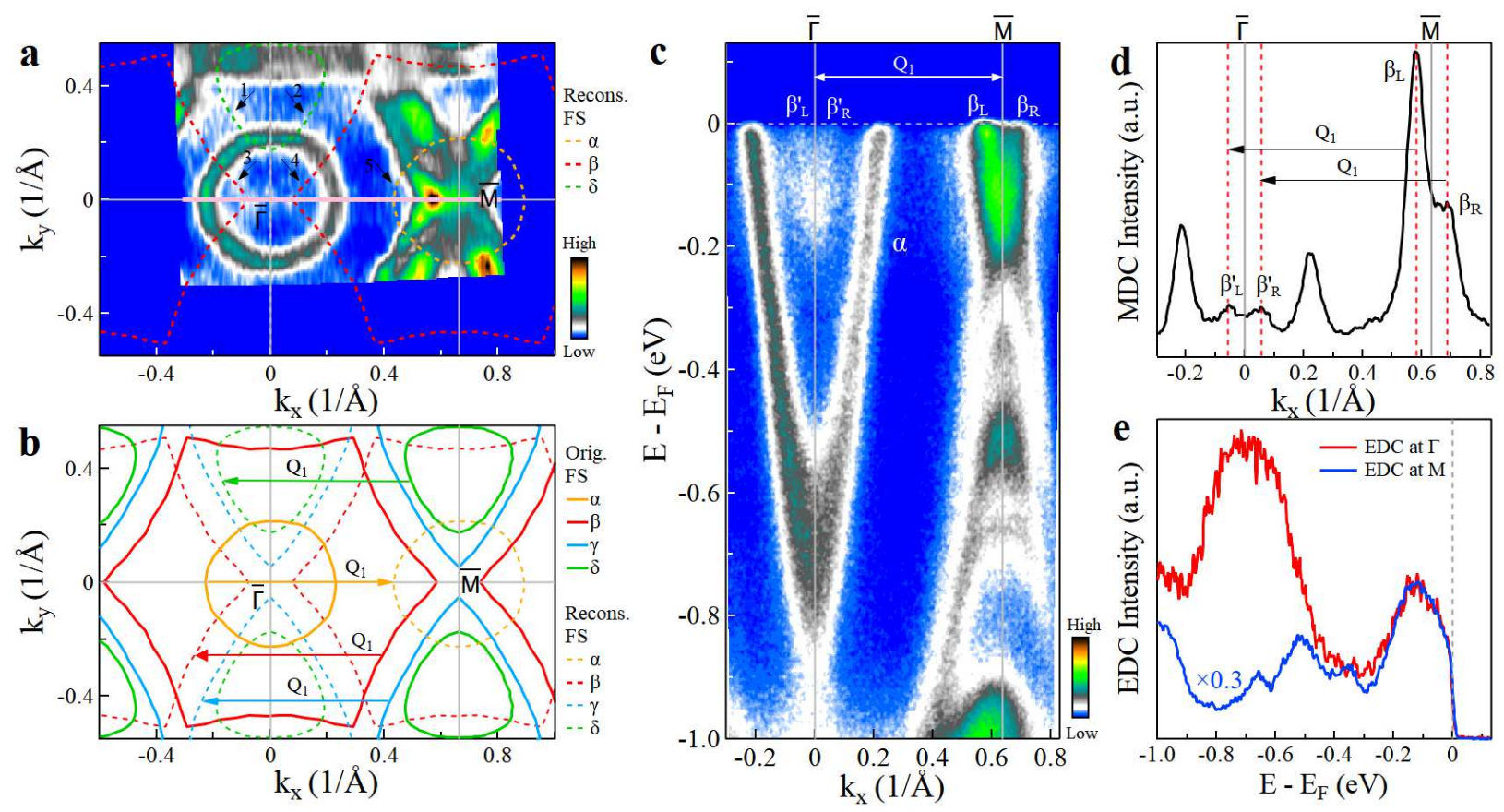

FIG. 2. Evidence of electronic structure reconstruction in $\mathbf{K V}_{3} \mathbf{S b}_{5}$. a, Fermi surface mapping of $\mathrm{KV}_{3} \mathrm{Sb}_{5}$ at $20 \mathrm{~K}$ in the $\mathrm{CDW}$ phase. In addition to the original Fermi surface, some extra weak features can be observed as marked by arrows and guided by the dashed lines that are reconstructed Fermi surface due to the CDW wavevector $Q_{1}$. b, Schematic of the reconstructed Fermi surface of $\mathrm{KV}_{3} \mathrm{Sb}_{5}$ due to $\mathrm{CDW}$ wavevector $Q_{1}$. The solid lines represent the original Fermi surface sheets. The reconstructed Fermi surface sheets (dashed lines) are obtained by shifting the original Fermi surface with a wavevector $\pm Q_{1}$. c, Band structure measured along the $\bar{\Gamma}-\bar{M}$ high-symmetry direction at $20 \mathrm{~K}$. The location of the momentum cut is marked as a solid pink line in (a). Around $\bar{\Gamma}$ just below the Fermi level, some extra bands can be observed. d, The momentum distribution curve (MDC) at the Fermi level from the band structure in (c). Two MDC peaks $\left(\beta_{L}\right.$ and $\left.\beta_{R}\right)$ can be observed around $\bar{M}$, and another two MDC peaks $\left(\beta_{L}^{\prime}\right.$ and $\left.\beta_{R}^{\prime}\right)$ can be observed around $\bar{\Gamma}$. The separation between $\beta_{L}$ and $\beta_{L}^{\prime}\left(\beta_{R}\right.$ and $\left.\beta_{R}^{\prime}\right)$ corresponds to the reconstruction wavevector $Q_{1}$. e, The photoemission spectra (energy distribution curves, EDCs) measured at $\bar{\Gamma}$ and $\bar{M}$ points in (c). They show similar EDC lineshape in the low binding energy region $\left(E_{B}<0.3 \mathrm{eV}\right)$. 

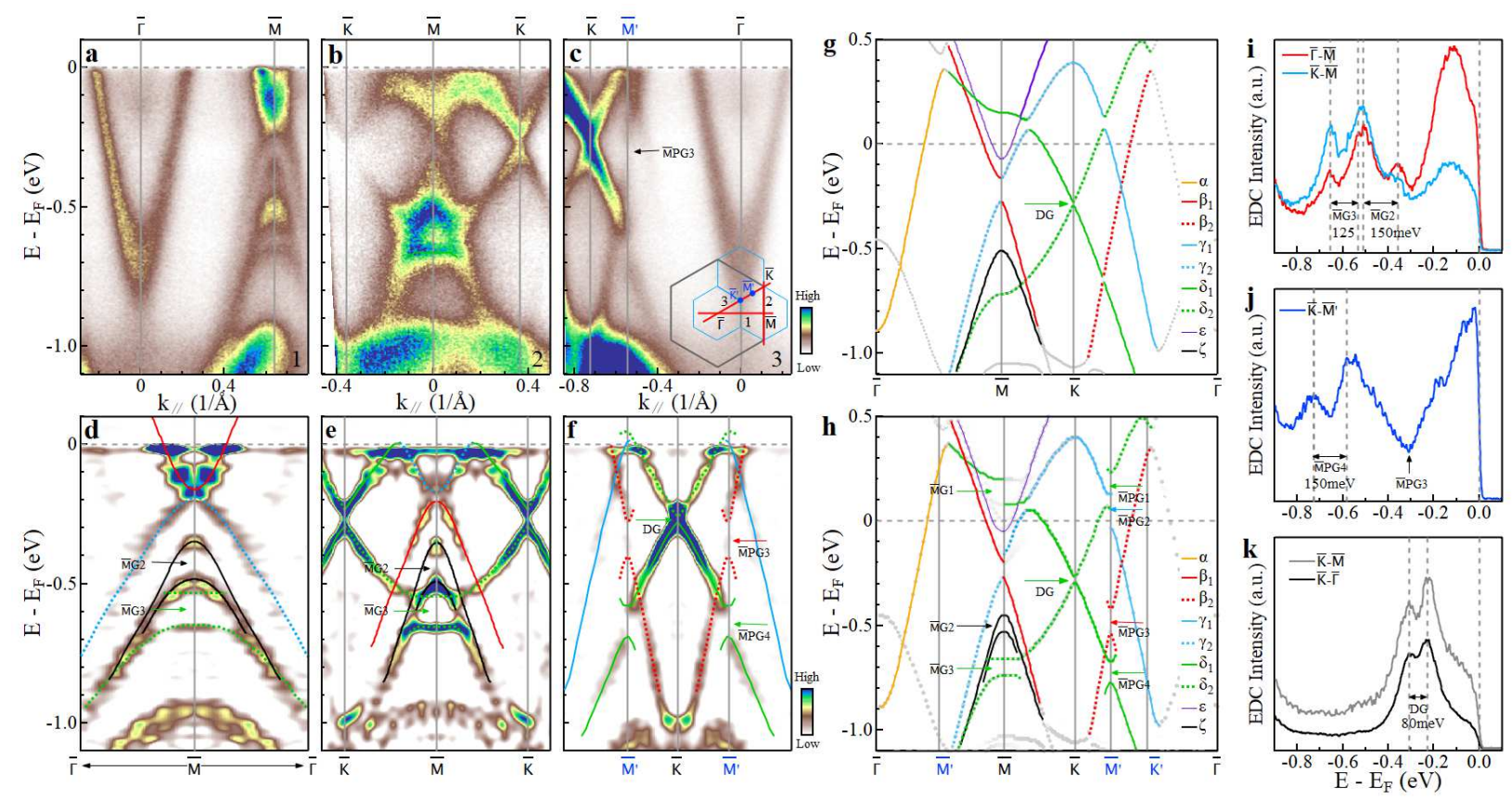

FIG. 3. CDW-induced band splitting and gap opening in the measured band structures of $\mathrm{KV}_{3} \mathrm{Sb}_{5}$ at $20 \mathrm{~K}$ and their comparison with band structure calculations. a-c, Band structures measured along the $\bar{\Gamma}-\bar{M}$ (a), $\bar{K}-\bar{M}-\bar{K}$ (b) and $\bar{K}-\bar{\Gamma}$ (c) high-symmetry directions, respectively. The locations of the momentum cuts, 1, 2 and 3 for (a), (b) and (c), respectively, are shown in the inset of (c). d-f, Detailed band structures around $\bar{M}$ and $\bar{K}$ points measured along $\bar{\Gamma}-\bar{M}(\mathrm{~d}), \bar{K}-\bar{M}-\bar{K}$ (e) and $\bar{K}-\bar{\Gamma}$ (f) directions, respectively. These are second derivative images obtained from the band structures in a-c,. The measured band structures are indicated by guide lines and the associated CDW gaps and SOC gap are also marked. g, Calculated band strcture of $\mathrm{KV}_{3} \mathrm{Sb}_{5}$ with pristine crystal structure in Fig. 1a at $\mathrm{k}_{z}=0.5$ with SOC. $\mathbf{h}$, The calculated band structures of $\mathrm{KV}_{3} \mathrm{Sb}_{5}$ with reconstructed $\mathrm{TrH}$ crystal structure in Fig. $1 \mathrm{~b}$ at $\mathrm{k}_{z}=0.5$ with SOC. In addition to the original high-symmetry points $\bar{\Gamma}, \bar{M}$ and $\bar{K}$, new high-symmetry points from the reconstructed Brillouin zone (Fig. 1c), $\bar{M}^{\prime}$ and $\bar{K}^{\prime}$, are marked. Three CDW gaps open at $\bar{M}$ : $\bar{M} \mathrm{G} 1, \bar{M} \mathrm{G} 2$ and $\bar{M} \mathrm{G} 3$ and four CDW gaps open at $\bar{M}^{\prime}: \bar{M} \mathrm{PG} 1, \bar{M} \mathrm{PG} 2, \bar{M} \mathrm{PG} 3$ and $\bar{M} \mathrm{PG} 4$. The SOC gap opening at the Dirac point at $\bar{K}$ is marked by DG. i, EDCs at $\bar{M}$ from band structures in (a) and (b). j, EDC at $\bar{M}^{\prime}$ from band structure in (c). The CDW gap size is measured by the separation between related EDC peaks. k, EDCs at $\bar{K}$ from band structures in (b) and (c). 

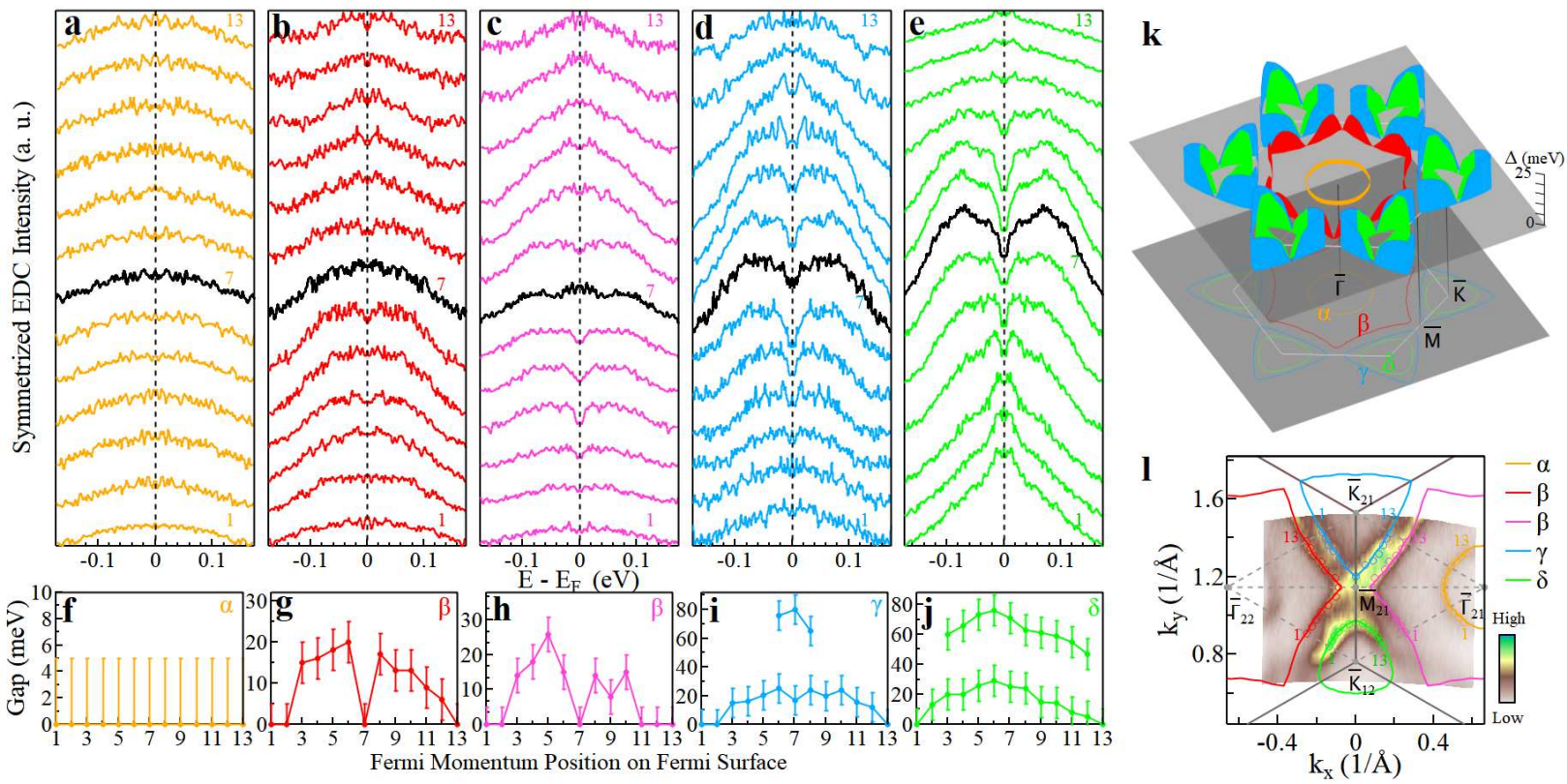

FIG. 4. Fermi surface- and momentum-dependent CDW gaps of $\mathrm{KV}_{3} \mathrm{Sb}_{5}$ measured at

5 K. a-e, Symmetrized EDCs along the Fermi surface sheets $\alpha$ (a), $\beta$ (b and c), $\gamma(\mathrm{d})$ and $\delta$ (e). The corresponding Fermi momentum positions are marked in (l) by numbers on each Fermi surface sheet. $\mathbf{f}-\mathbf{j}$, CDW gap size as a function of momentum on the Fermi surface $\alpha$ (f), $\beta$ (g and h), $\gamma$ (i) and $\delta(\mathrm{j})$. The gap size is obtained by picking the peak positions in the symmetrized EDCs in (a-e). When multiple peaks are observed in (i) and $(\mathrm{j})$, the position of the higher binding energy peak is also extracted. k, Three-dimensional plot of the Fermi surface-dependent and momentumdependent $\mathrm{CDW}$ gaps in $\mathrm{KV}_{3} \mathrm{Sb}_{5}$. 1, High-resolution Fermi surface mapping of $\mathrm{KV}_{3} \mathrm{Sb}_{5}$ at $5 \mathrm{~K}$. The observed four Fermi surface sheets $\alpha, \beta, \gamma$ and $\delta$ are marked. 

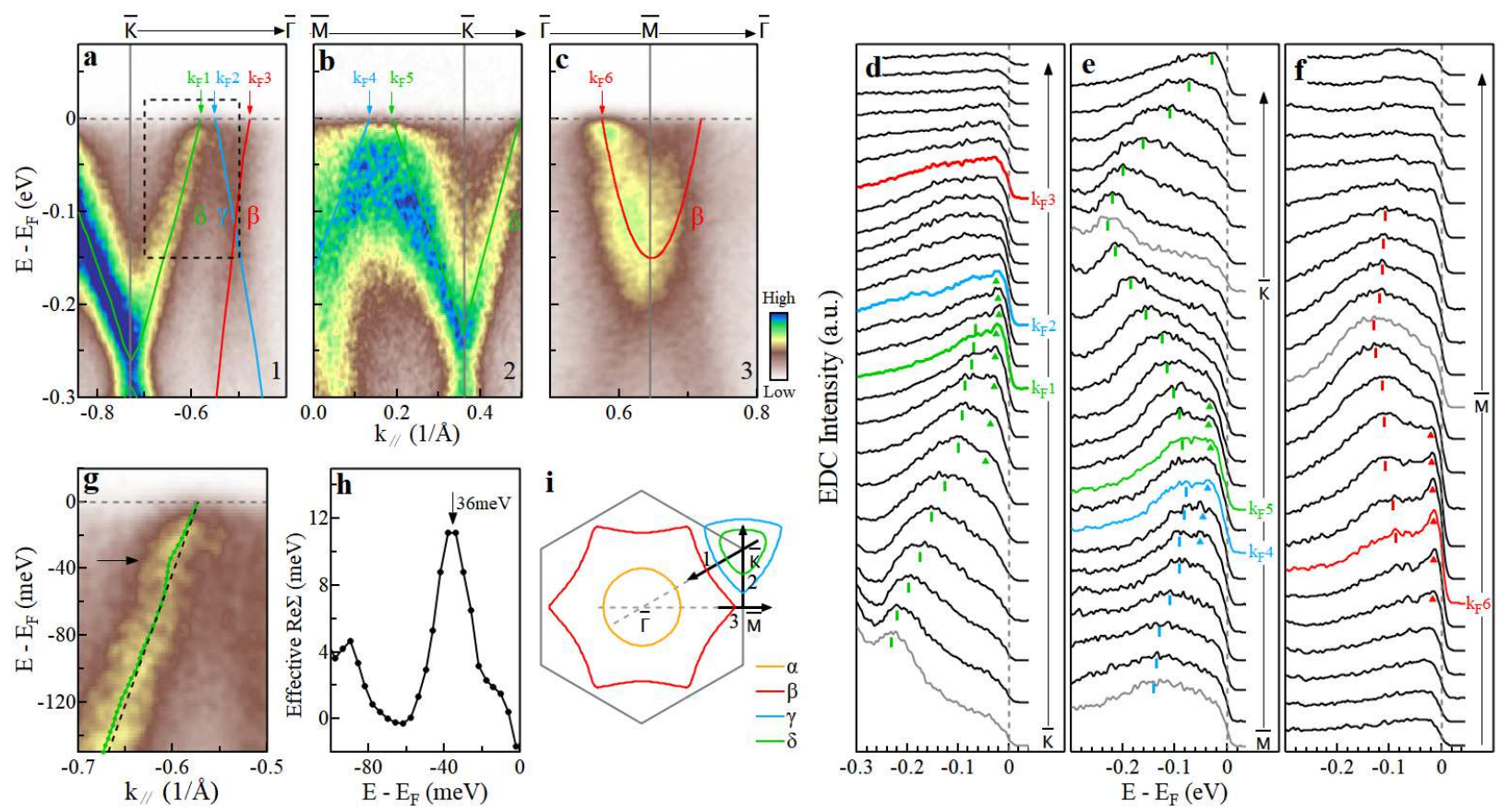

FIG. 5. Electron-phonon coupling in $\mathbf{K V}_{3} \mathbf{S b}_{5}$. a-c, Detailed band structures along $\bar{K}-\bar{\Gamma}$ (a), $\bar{M}-\bar{K}$ (b) and $\bar{\Gamma}-\bar{M}-\bar{\Gamma}$ (c) directions, respectively, measured at $20 \mathrm{~K}$. The analysis of these band structures is shown in Fig. S4 in Supplementary Materials. The locations of the momentum cuts, 1, 2 and 3 for (a), (b) and (c), respectively, are shown in (i). The Fermi momenta of the $\beta, \gamma$ and $\delta$ bands are marked by arrows and labeled by $\mathrm{k}_{F} 1 \sim \mathrm{k}_{F} 6$. d-f, The corresponding EDCs for the band structures in (a)-(c), respectively. Peak-dip-hump structure can be observed near $\mathrm{k}_{F} 1, \mathrm{k}_{F} 2, \mathrm{k}_{F} 4$, $\mathrm{k}_{F} 5$ and $\mathrm{k}_{F} 6$. The EDC peaks are marked by triangles while the humps are marked by bars. $\mathrm{g}$, Expanded view of the $\delta$ band inside the dashed box in (a). The MDC fitted dispersion is shown by green line and the dashed black line represents an empirical bare band. $\mathbf{h}$, Effective real part of the electron self-energy $(\Sigma)$ extracted from $(\mathrm{g})$. It shows a peak at $\sim 36 \mathrm{meV}$. i, Schematic of the Fermi surface and the locations of the momentum cuts 1, 2 and 3 for the band structures in (a), (b) and (c), respectively. 


\section{Supplementary Files}

This is a list of supplementary files associated with this preprint. Click to download.

- 20210715KV3Sb5SI.pdf 